\title{
Discovery of rare variants implicated in schizophrenia using next-generation sequencing
}

\author{
Raina Rhoades, Fatimah Jackson, Shaolei Teng \\ Department of Biology, Howard University, Washington, DC 20059, USA. \\ Correspondence to: Dr. Shaolei Teng, Department of Biology, Howard University, Washington, DC 20059, USA. \\ E-mail: shaolei.teng@howard.edu
} How to cite this article: Rhoades R, Jackson F, Teng S. Discovery of rare variants implicated in schizophrenia using next-generation
sequencing. J Trans/Genet Genom 2019;3:1. https://doi.org/10.20517/jtgg.2018.26

Received: 31 Aug 2018 First Decision: 20 Nov 2018 Revised: 5 Dec 2018 Accepted: 6 Dec 2018 Published: 20 Jan 2019

Science Editor: Sheng-Ying Qin Copy Editor: Cui Yu Production Editor: Huan-Liang Wu

\begin{abstract}
Schizophrenia is a highly heritable psychiatric disorder that affects $1 \%$ of the population. Genome-wide association studies have identified common variants in candidate genes associated with schizophrenia, but the genetics mechanisms of this disorder have not yet been elucidated. The discovery of rare genetic variants that contribute to schizophrenia symptoms promises to help explain the missing heritability of the disease. Next generation sequencing techniques are revolutionizing the field of psychiatric genetics. Various statistical approaches have been developed for rare variant association testing in case-control and family studies. Targeted resequencing, whole exome sequencing and whole genome sequencing combined with these computational tools are used for the discovery of rare genetic variations in schizophrenia. The findings provide useful information for characterizing the rare mutations and elucidating the genetic mechanisms by which the variants cause schizophrenia.
\end{abstract}

Keywords: Rare variant, schizophrenia, next-generation sequencing, rare variant association study, targeted resequencing, whole genome sequencing, whole exome sequencing

\section{INTRODUCTION}

Schizophrenia (SCZ) is a serious psychiatric disorder that affects $1 \%$ of Americans ${ }^{[1]}$, and over 23 million cases are estimated in the world. The financial costs of the disease have been estimated to be $\$ 62.7$ billion in the United States ${ }^{[2]}$. The economic burden of SCZ, including both direct costs and indirect costs, is estimated to be $\$ 155.7$ billion dollars annually ${ }^{[3]}$. People with SCZ may experience hallucinations, delusions, disorganized speech and social withdrawal ${ }^{[4]}$. Psychiatric comorbidities in these patients include depression, anxiety and cognitive deficits ${ }^{[5]}$. The disease has great impact on the quality of life for the patients and their

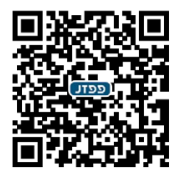


families. Approximately $40 \%$ of SCZ patients attempt suicide and are at eight-fold higher risk than the general population.

The underlying biological mechanisms of SCZ remain elusive. The disease is known to be accompanied by dysfunction in neurotransmission; morphological changes in prefrontal cortex, hippocampus, and striatum; and alterations in the signaling between brain regions as well ${ }^{[6-9]}$. Some environment factors such as childhood trauma can increase the risk for $\mathrm{SCZ}^{[10]}$. The twin and family studies suggest that the genetic heritability is the most significant risk factor for developing the disease. For example, monozygotic twins are more than twice as likely to be diagnosed with SCZ than dizygotic twins ${ }^{[11]}$. Determining the genetics of SCZ and complex disorders is further complicated by evolutionary genetic theory. Though it would seem that common complex heritable disorders, should be phased out through natural selection, several plausible theories have been used to explain this paradox, including balancing selection, heterozygote advantage, pleiotrophy, and polygenic models ${ }^{[12]}$. The polygenic model seems to be supported throughout the literature, however determining the precise number of loci that contribute to a particular complex disorders or traits has been challenging ${ }^{[12]}$. Many genetic approaches have been applied to the investigation of genetic variants that play an important role in the development of $\mathrm{SCZ}^{[12]}$. Genome-wide association studies (GWAS) have identified single nucleotide polymorphisms (SNPs) associated with increased susceptibility in SCZ ${ }^{[13,14]}$. The loci revealed by GWAS have diverse biological roles including neurotransmission, inflammatory response, glucose metabolism, and cell adhesion ${ }^{[15]}$. Several studies have demonstrated weak associations between common variants and major psychiatric disorders such as SCZ, bipolar disorder (BD) and major depressive disorder $(\mathrm{MDD})^{[16]}$. However, most of the common variants have small or moderate effects on the disease risk, and a large proportion of the heritability remains unexplained.

Rare variants, usually defined as alleles with a minor allele frequency (MAF) $<1 \%$ in population, have been demonstrated to contribute to the missing heritability in SCZ. The use of next-generation sequencing (NGS) technologies like targeted resequencing, whole exome sequencing (WES) and whole genome sequencing (WGS) have allowed scientists to identify the rare variants and estimate the deleterious mutation load to investigate the contribution of rare mutation to the complex diseases ${ }^{[17]}$. In a SCZ study, WES for 2,536 cases and 2,543 controls showed enrichment of rare disruptive variants in genes related to calcium channels and the fragile $\mathrm{X}$ mental retardation protein ${ }^{[18]}$. Identifying rare variants is important for prioritizing potential pathogenic targets for diagnosis and drug discovery. This review will focus on the development of rare variant association tools and recent discoveries for investigating rare variants implicated in SCZ using NGS.

\section{COMMON VARIANTS VS. RARE VARIANTS}

\section{Common vs, rare variant hypotheses for complex diseases}

There are two contemporary hypotheses concerning the genetic contribution of sequence variants for common complex disorders such as cancer and $\mathrm{SCZ}^{[19]}$. The "Common Disease, Common Variant" (CD-CV) hypothesis argues that common diseases are associated with the common genetic variants. It means that a few variants with high frequencies in the population are the major contributors for a common disease with complex traits. However, the common variant can only account for a portion of heritability of many genetic disorders ${ }^{[20]}$. One investigation demonstrated that the association of common variants with many disorders and complex traits only accounted for approximately $30 \%$ of the heritability in $\mathrm{SCZ}^{[21]}$. This finding implies that the missing heritability is due to undiscovered common variants and rare disruptive variants. To address the missing heritability issue, the "Common Disease, Rare Variant" hypothesis suggests that multiple rare variants with relatively high penetrance contribute to the genetic component of common diseases. These genetic variations are usually found within less than one percent of the population. Some studies have demonstrated that rare variants could account for a substantial percentage of the missing heritability in complex diseases and phenotypes ${ }^{[22]}$. Determining the precise sources of missing heritability is a necessary hurdle to understand the etiology of neuropsychiatric disorders. 


\section{GWAS and common variants}

The CD-CV hypothesis provides the scientific paradigm for GWAS, which is a powerful tool for understanding the etiology of complex disorders ${ }^{[23]}$. GWAS usually applies SNP arrays to identify the target genes that may be involved with common diseases from the entire genome ${ }^{[24]}$. This methodology depends heavily on assessing the correlation of MAFs of different variants and determining whether they are correlated with a set of traits ${ }^{[25]}$. GWAS has elucidated many common variants in complex illnesses. For example, a GWAS study for Crohn's disease identified over 30 candidate variants, and also demonstrated that variants in $I L K 23 R$ and $I L 12 B$ are also associated with psoriasis and other autoimmune disorders ${ }^{[26]}$.

GWAS has been used to identify many common variants associated with SCZ. Betcheva et al. ${ }^{[27]}$ performed a GWAS analysis to screen 554,496 SNPs in 188 SCZ patients and 376 controls from Bulgaria. One SNP, rs7527939, in the HHAT gene demonstrated a significant association with SCZ with an odds ratio of 2.63. Previous work has shown that the microstructure of white matter is altered in the brains of SCZ, specifically in the left and right anterior cingulate, left and right posterior cingulate, the inferior parietal cortices than was present in unaffected controls. Univariate association analysis showed that one variant upstream of the CXCR7 gene, was associated with a reduction in white matter, and a multivariate analysis revealed an association with the SORCS 1 gene ${ }^{[28]}$, which lends support to the polygenic etiology of SCZ ${ }^{[29]}$. Many of the genes affected by these common variants are known to play a role in many important cellular functions, such as mitotic arrest, signal transduction, voltage dependent calcium receptors as well growth and differentiation cells ${ }^{[30]}$. GWAS have also been instrumental in elucidating pathways that are enriched with SNPs associated with SCZ. These pathways include serotonergic signaling, ubiquitin mediated proteolysis, hedgehog signaling, adipocytokine signaling, and renin secretion. It is interesting to note that the SNPs that were enriched in the aforementioned pathways were all found in regulatory regions ${ }^{[31]}$.

There are limitations for using GWAS to investigate the role of sequence variants in disease. GWAS studies are based around the concept of linkage disequilibrium (LD), whereby alleles within a particular locus are generally more closely related than the alleles that are located more distantly. The strength of the LD is dependent on the frequency at which alleles appear within the population. The greater the allelic frequency or the more common the variation is, the stronger the association or LD. Thus, many of the SNPs identified as being associated with a particular trait are not likely to be causal due to $\mathrm{LD}^{[32]}$. Increasing the sample size significantly, however, will result in the selection of several common variants of small effect. Meanwhile, rare variants will be masked by or undetected by GWAS because of low statistical power, caused by small number of cases, low allelic frequencies, low prevalence rates, etc. ${ }^{[33,34]}$. In addition, sample size has an important effect on the results of common variant analysis in GWAS studies, such that small sample sizes will often result in the identification of few variants with large effects ${ }^{[35]}$.

\section{NGS and rare variants}

Rare variants are generally not detectable by GWAS because of their low frequencies which makes detecting them much more difficult than common variants. Multiple NGS technologies have been developed to identify the rare variants including single nucleotide variants (SNVs) and copy number variations (CNVs). Target resequencing takes advantage of using multiple probes or multiplexed PCR techniques to enrich specific regions of genes and is far less costly than using custom arrays ${ }^{[36]}$. WES uses targeted gene panels to sequence the coding regions, approximately $2 \%$ of the human genome. It provides a less expensive way to search for sequence variants throughout the genome ${ }^{[37]}$. Finally, WGS can be used to search for variants throughout the entire genome ${ }^{[37]}$. It also captures the non-coding regulatory regions ${ }^{[38]}$, which are important for gene expression. However, prior knowledge regarding the functional relevance of sequences found in non-coding regions is necessary in order to collapse or aggregate these rare variants in a meaningful way ${ }^{[39]}$. 


\section{Common Variant}

\section{SNP-level}

$\rightarrow>>$ TCTGACGTGGC $->->>->$ TTGCATACAGA $\rightarrow->>$ ATAACTGAGTA $\rightarrow->$ $\rightarrow>$ TCTGACGTGGC $\rightarrow->>>->$ TGGATACAGA $\rightarrow>->$ ATAACAGAGTA $\rightarrow>>$ $\rightarrow>$ TCTGACGTGGC $->>>->$ TTGCATACAGA $\rightarrow>>>$ ATAACTGAGTA $>>>>$ $\rightarrow>$ TCTGACGTGGC $->->>>>$ TTGCATACAGA $\rightarrow->>$ ATAACTGAGTA $>->$ u $\rightarrow>>$ TCTGACGTGGC $>>->->$ TTGCATACAGA $\rightarrow->>$ ATAACTGAGTA $\rightarrow->$

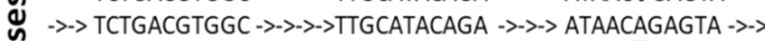
J $\gg>$ TCTGACGTGGC $->>->>$ TTGCATACAGA $\gg->>$ ATAACTGAGTA $>->$ $\rightarrow->$ TCTGACGTGGC $->>->>>$ TTGCATACAGA $\rightarrow>>>$ ATAACTGAGTA $\rightarrow->$ $\rightarrow>$ TCTGACGTGGC $\rightarrow>>->>$ TTGCATACAGA $\rightarrow>>>$ ATAACAGAGTA $\rightarrow>>$ $\rightarrow->$ TCTGACGTGGC ->->->->TTGCATACAGA $->->->$ ATAACTGAGTA $->->$

$\rightarrow>$ TCTGACGTGGC $>->>->$ TGCATACAGA $\rightarrow>>->$ ATAACAGAGTA $\rightarrow>>$ $\rightarrow>$ TCTGACGTGGC $->>->>$ TTGCATACAGA $\gg->>$ ATAACAGAGTA $>>>$ $\rightarrow>$ TCTGACGTGGC $>>>->>$ TTGCATACAGA $->>->$ ATAACAGAGTA $\rightarrow>>$

$\rightarrow>$ TCTGACGTGGC $\rightarrow->->>$ TTGCATACAGA $\rightarrow->>$ ATAACTGAGTA $\rightarrow->$ $\rightarrow>$ TCTGACGTGGC $>>>->>$ TTGCATACAGA $>->->$ ATAACAGAGTA $>>>$ $\rightarrow>>$ TCTGACGTGGC $>>>->>$ TTGCATACAGA $\rightarrow>>->$ ATAACTGAGTA $\rightarrow>->$ $\rightarrow>$ TCTGACGTGGC $\rightarrow>>->$ TTGCATACAGA $\rightarrow>->$ ATAACAGAGTA $\gg->$ $\rightarrow>$ TCTGACGTGGC $->->->$ TTGCATACAGA $\rightarrow->>$ ATAACAGAGTA $->>$ $\rightarrow>$ TCTGACGTGGC $->->>->$ TTGCATACAGA $\gg->>$ ATAACAGAGTA $>->$ $\rightarrow>$ TCTGACGTGGC $\rightarrow>>->>$ TTGCATACAGA $\rightarrow->>$ ATAACAGAGTA $\rightarrow>>$

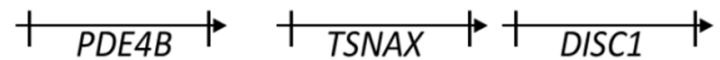

\section{Rare Variant}

GeneSet-level

Figure 1. Statistical approaches for common and rare variant association studies. Common variant association studies can detect the association at the SNP-level. However, rare variant association analysis requires collapsing of variants into groups such as gene sets or genes. SNP: single nucleotide polymorphism

Rare causal variants can be hard to identify precisely because they are so uncommon due to evolution placing negative selection pressure on alleles with deleterious effects on fitness ${ }^{[40]}$. It is worth noting that complex disorders like SCZ seem to have escaped this negative selection pressure, suggesting that these disorders are polygenic or arising from multiple rare genetic variations. Although experimental strategies can help make the identification of rare variants easier, ultimately the application of computational statistical methods is required. NGS results in poor signal to noise ratio when used for detecting rare variants. Therefore, it is important to use targeted enrichment methods such as targeted resequencing or WES, along with computational tools such as annotation databases and softwares and statistical methods ${ }^{[38]}$. Even when these methods are applied, the effect sizes of rare variants are expected to be moderate to weak when compared to common variants. So determining the sequencing depth is an important decision in terms of experimental design, as larger samples and fewer reads per base might be more desirable than high depth read of a few cases and controls ${ }^{[40]}$. Determining how one should annotate sequences is also critical to the analysis of rare variants, and many tools have been designed to identify different types of mutations and structural variations ${ }^{[41]}$.

\section{STATISTICAL TOOLS FOR RARE VARIANTS ASSOCIATION STUDIES}

Different statistical approaches have been developed to investigate the associations between common and rare variants and genetic disorders [Figure 1]. The single marker testing is usually applied in GWAS to identify the common variants associated with complex disorders. In this approach, the genotypes of each SNP are coded and their effects are computed based on the number of variants in case and controls using the statistical tests such as Student's $t$-test and Fisher's exact test ${ }^{[2]}$. The rare variant association study (RVAS) methodologies require much larger samples sizes than GWAS because the signal is lower overall for rare variants than for common variants. Due to low statistical power, it is not possible to determine the effect size of single rare variants on complex diseases or phenotypes ${ }^{[43]}$. Therefore, the association approach was developed by grouping the rare variants across a genomic region, a gene or multiple genes ${ }^{[40]}$. Multiple 
marker testing evaluates the effects of multiple rare variants to determine whether they are associated with the disease or trait under investigation. The outcome of either test will depend greatly on whether the disease is the result of a single common variant or of multiple rare variants. Here we review the approaches that have been applied to the investigation of rare variants in genetic disorders in general, and how these tools have been employed for analyzing NGS data [Table 1].

\section{Approaches for case-control studies}

RVAS approaches use statistical methods to combine the effects of rare variants to strengthen signals. The burden test is carried out by collapsing variants in a gene or functional region into a single score, then the association between the collapsed score and the phenotype is computed. The collapsing of the variants is accomplished either through the selection of a threshold or by the use of permutation tests, which require a lot of computational power ${ }^{[4]}$. Collapsing of variants into a single score results in each variant being treated as though it has the same effect on the phenotype. One way to test for the varying effects of rare variants on phenotype is to use the Multi-phenotype Analysis of Rare Variants (MARV) test. This is a type of burden analysis that utilizes multi-phenotype analysis. MARV calculates MAF at rare variants within a region of the genome. It then performs a linear regression to associate phenotype or combinations of phenotypes with the MAF for each variant ${ }^{[44,45]}$. The cohort allelic sums test is a burden test that collapses variants into a single score and can identify genes that carry one or more risk alleles. The score indicates the presence or absence of a minor allele, which is then tested for its association with a phenotype using univariate analysis ${ }^{[46]}$. Meanwhile, the combined multivariate and collapsing method combines the collapsing of rare variants with multivariate analysis of both collapsed rare variants and uncollapsed common variants ${ }^{[47]}$.

Note that the burden test assumes that all rare variants contribute the disease in the same direction. However, most rare variants have small effects on disease. In addition, some variants are disease-causing mutations, and some are protective variants. To address this scenario, the variance-component tests were developed. The $\mathrm{C}$-alpha test can be used to determine the directionality of an effect of multiple variants on a phenotype. The generalized C-alpha test generates scores, based on summary statistics, which evaluates the increasing and or decreasing effects of multiple variants on a phenotype based on the Gaussian distribution. The phenotype can be binary or quantitative. The C-alpha test can be used on large population datasets or on smaller samples, such as familial studies. The C-alpha test operates on three basic assumptions: the number of variants, strength and independence of the effects, and the assumption that the variants are normally distributed $^{[48]}$.

The Sequence Kernel Association Test (SKAT) is a type of supervised machine learning, that evaluates each variant based on a $P$ value and then weights each variant based on a linear or logistical regression model ${ }^{[49]}$. One advantage of SKAT is that it allows for the detection of interactions between variants ${ }^{[50]}$. This method also allows for the epistatic effects to be revealed. However, in the case where many rare variations are in a particular region and have a similar effect on the phenotypes, the optimal unified test, called SKAT-Optimal Unified Test (SKAT-O), can be used. Rather than using collapsed scores, SKAT-O utilizes the minimum $P$ values from different kernels, which include correlation effects ${ }^{[50]}$. The SKAT-O program, due to its power. When sample sizes are insufficient to generate accurate $P$ values, the adaptive procedure-SKAT (AP-SKAT) can be used. This software is similar to SKAT, except that it "adaptively stops" the permutation test when the $P$-value is outside of the confidence interval of the $P$ value that would be predicted using a binomial distribution. This procedure can be used to reduce the risk of obtaining a type I error ${ }^{[51]}$. AP-SKAT is more efficient in terms of computation than either SKAT or SKAT-O ${ }^{[4]}$. To investigate the dataset with fewer samples than required by SKAT, exact variance component tests can be used ${ }^{[52]}$. These tests minimize type 1 errors and in small samples, these types of tests identify more genes associated with polygenic traits that are identified by SKAT. Gene association with multiple traits is a variation of the sequence kernel association, called kernel distance covariance. This test uses non-parametric tests to test the association between rare 
Table 1. Rare variants association tools

\begin{tabular}{|c|c|c|c|}
\hline Tools & URL & Description & References \\
\hline \multicolumn{4}{|c|}{ Burden test tools for case-control studies } \\
\hline MARV & https://github.com/ImperialStatGen/MARV & $\begin{array}{l}\text { Collapses rare variants into } \\
\text { genomic region and performs } \\
\text { analyses based on the proportion } \\
\text { of minor alleles in rare variants }\end{array}$ & Kaakinen et al. ${ }^{[45]}$ \\
\hline CAST & https://rdrr.io/cran/AssotesteR/man/CAST.html & $\begin{array}{l}\text { Variants are collapsed into a single } \\
\text { variable and tested frequency } \\
\text { association with the phenotype in } \\
\text { question using univariate analysis }\end{array}$ & $\begin{array}{l}\text { Morgenthaler and } \\
\text { Thilly }^{[46]}\end{array}$ \\
\hline CMC & http://varianttools.sourceforge.net/Association/CMC & $\begin{array}{l}\text { A method that combines } \\
\text { collapsing with multivariate } \\
\text { analysis }\end{array}$ & Li and Leal ${ }^{[47]}$ \\
\hline \multicolumn{4}{|c|}{ Variance component test tools for case-control studies } \\
\hline C-Alpha & $\begin{array}{l}\text { https://cran.r-project.org/web/packages/AssotesteR/ } \\
\text { AssotesteR.pdf }\end{array}$ & $\begin{array}{l}\text { Analyzes the distribution of rare } \\
\text { variants }\end{array}$ & Neale et al. ${ }^{[48]}$ \\
\hline SKAT & https://www.hsph.harvard.edu/skat/ & $\begin{array}{l}\text { Supervised machine learning test } \\
\text { for the effect of multiple variants } \\
\text { within a gene get or region }\end{array}$ & Wuet al. ${ }^{[49]}$ \\
\hline SKAT-O & http://www.hsph.harvard.edu/ ${ }^{x}$ lin/software.html & $\begin{array}{l}\text { Uses the values from different } \\
\text { kernels, which include correlation } \\
\text { effects }\end{array}$ & Lee et $a l^{[50]}$ \\
\hline AP-SKAT & http://nagasakilab.csml.org/data/aSKAT.zip & $\begin{array}{l}\text { "Adaptively stops" the } \\
\text { permutation test when the } \\
P \text {-value is outside the confidence } \\
\text { interval predicted by the binomial } \\
\text { distribution }\end{array}$ & Hasegawa et al.. ${ }^{[51]}$ \\
\hline Exact VCTest & https://github.com/Tao-Hu/VarianceComponentTest.jl & $\begin{array}{l}\text { Can be used with fewer samples } \\
\text { than SKAT }\end{array}$ & Zhou et $a l^{[52]}$ \\
\hline GAMuT & https://epstein-software.github.io/GAMuT/ & $\begin{array}{l}\text { Uses a non-parametric test } \\
\text { to determine the association } \\
\text { between rare variants and } \\
\text { phenotypes }\end{array}$ & Chiu et $a .^{[53]}$ \\
\hline \multicolumn{4}{|c|}{ Tools for family studies } \\
\hline MONSTER & http://www.stat.uchicago.edu/ mcpeek/software/index.html & $\begin{array}{l}\text { Extension of SKAT-O that can be } \\
\text { used to correct for kinship }\end{array}$ & Jiang and Mcpeek ${ }^{[55]}$ \\
\hline famSKAT & https://www.hsph.harvard.edu/han-chen/2014/07/31/famskat/ & $\begin{array}{l}\text { A statistical strategy that also } \\
\text { uses sequence kernel association } \\
\text { to evaluate rare variants in } \\
\text { samples that contain related } \\
\text { individuals }\end{array}$ & Chen et $a l^{[56]}$ \\
\hline pVAAST & http://www.hufflab.org/software/pvaast/ & $\begin{array}{l}\text { Be used to evaluate associations } \\
\text { between rare variants and } \\
\text { phenotypes }\end{array}$ & Hu et $a .^{[57]}$ \\
\hline GEE-KM & https://github.com/xfwang/ & $\begin{array}{l}\text { Analyzes binary traits in family } \\
\text { studies }\end{array}$ & Wang et $a l^{[58]}$ \\
\hline RVFAM & https://cran.r-project.org/web/packages/RVFam/index.html & $\begin{array}{l}\text { SNP for associations with either } \\
\text { continuous, binary, or survival } \\
\text { phenotypes in familial sequencing } \\
\text { studies }\end{array}$ & Chen and Yang ${ }^{[59]}$ \\
\hline FBAT & https://sites.google.com/view/fbat-web-page & $\begin{array}{l}\text { A burden test with a variance } \\
\text { component that can be used for } \\
\text { rare variant association testing } \\
\text { within extended families }\end{array}$ & Wang et al. ${ }^{[60]}$ \\
\hline FARVAT & http://healthstat.snu.ac.kr/software/farvat/ & $\begin{array}{l}\text { Collapses variants into weighted } \\
\text { sum statistics that are tested for } \\
\text { association using multivariate, } \\
\text { regression, or linear combination } \\
\text { analyses }\end{array}$ & Choi et al. ${ }^{[61]}$ \\
\hline
\end{tabular}

MARV: Multi-phenotype Analysis of Rare Variants; CAST: cohort allelic sums test; CMC: combined multivariate and collapsing; SKAT: sequence kernel association test; Exact VCTest: exact variance component tests; GAMuT: gene association with multiple traits; MONSTER: Minimum $P$-value Optimized Nuisance parameter Score Test Extended to Relatives; pVAAST: Pedigree Variant Annotation, Analysis, and Search Tool; GEE-KM: Kernel Machine Generalized Estimating Equations model; RVFAM: R package for rare variant association analysis with family data; SNP: single nucleotide polymorphism; FBAT: family-based association tests; FARVAT: family-based rare variant association test 
variants and multiple phenotypes ${ }^{[53]}$. Similarity and dissimilarity are assessed for both genotype and phenotype and a matrix is formed for each variable. Then, the similarity or dissimilarity matrices for each variable are tested for independence. The calculation of $P$-values does not require any permutations and the method can be utilized on WES or WGS ${ }^{[53]}$.

\section{Tools for family studies}

Family-based study designs are extremely advantageous to the study of rare variants because the frequency of rare alleles for a particular illness or disorder will be higher in a pedigree than among unrelated individuals ${ }^{[54]}$. Currently, there are very few rare variant analysis tools that are designed to find associations within sequences from family studies. The sampling of relatives in sequencing studies can help one to avoid sequencing errors in the analysis ${ }^{[55]}$. Therefore, the Minimum $P$-value Optimized Nuisance parameter Score Test Extended to Relatives (MONSTER) was developed. MONSTER is an extension of SKAT-O and tests for the association between rare variants and a phenotype, however, it can correlate data based on kinship ${ }^{[55]}$. It combines the SKAT model with a burden test model, where depending on the dataset presented $\rho$ will either be equivalent to zero, as in family-based SKAT (famSKAT), or equal to 1 as is the case with family-based burden test (famBT). famSKAT is a statistical strategy that uses sequence kernel association to evaluate rare variants in samples that contain related individuals ${ }^{[56]}$. FamBT is a burden analysis that can be used to evaluate associations between rare variants and phenotypes when samples contain kin. However, MONSTER is capable of adaptively switching between models, performing like either famSKAT or famBT depending on the data imported ${ }^{[5]}$.

A particular challenge in conducting a rare variant analysis of pedigree sequencing data is identifying de novo mutations ${ }^{[57]}$. Pedigree Variant Annotation, Analysis, and Search Tool is one of the tools that exists for rare variant analysis of familial data, it uses both association testing and the logarithm of odds (lod) scores to identify rare causal variants from familial data ${ }^{[57]}$. Fampipe is a pipeline that can be used to analyze rare variant data from association studies, the pipeline can calculate identity by descent scores as well as lod scores to identify regions that demonstrate association ${ }^{[54]}$. The pipeline has several modules capable of calculating allelic frequency, family-specific mutations and more. To analyze binary traits in familial based studies, the Kernel Machine Generalized Estimating Equations model (GEE-KM) was developed ${ }^{[58]}$. The Rare Variant association analysis with Family data (RVFam) package for R analyzes SNP for associations with either continuous, binary, or survival phenotypes in familial sequencing studies ${ }^{[59]}$. The family-based association tests $(\mathrm{FBAT})^{[60]}$ collapse variants using the sums of allele frequencies to generate test statistics that are weighted. These weighted summed stats are then tested for association with phenotypes using either multiple regression, linear regression, or linear combination analyses. Family-based Rare Variant Association Test ${ }^{[61]}$ is an extension of FBAT, a burden test with a variance component that can be used for rare variant association testing within extended families. The RVAS approaches can also be used to investigate rare and de novo noncoding variants in family studies. An analytical framework has been developed to investigate the de novo variations from WGS data in autism spectrum disorder (ASD) families ${ }^{[62]}$. The SNVs and indels are annotated and grouped by variant type, gene, species conservation, gene set, and regulatory region. The number of de novo mutations located in these regions in cases was compared to the number in sibling controls. Burden analyses are then performed to compute the significance of these comparisons. A similar procedure was used to detect the associations of de novo structural variants in different annotation groups. The authors analyzed rare variants in 519 ASD families and did not detect the significant association between rare de novo mutations in non-coding regions and ASD. However, they observed some biologically plausible associations that might warrant further investigation ${ }^{[62]}$.

\section{TARGET RESEQUENCING OF CANDIDATE GENES}

Targeted resequencing was developed to sequence the target genes or regions of interest ${ }^{[63]}$. The primary advantage of the technology is that they allow for more targeted sequencing of specific portions of the 
genome. Targeted resequencing can be used to identify rare variants in candidate genes associated with SCZ [Table 2]. One strategy is to sequence a few extreme cases and then compare the rare variants identified in cases with a few controls. The other strategy is to use a few extreme cases to identify rare variants in novel genes and perform a sequencing on a large cohort, targeting the potential candidate gene ${ }^{[64]}$.

\section{Disrupted-in-Schizophrenia 1}

Disrupted-in-Schizophrenia 1 (DISC1) is a candidate gene implicated in multiple major psychiatric disorders including SCZ, BD and MDD. It was originally reported in a linkage analysis of a translocation (1;11) (q42;q14) co-segregated with SCZ, BD, and MDD in a large Scottish pedigree ${ }^{[65]}$. Several additional independent studies have also reported associations between DISC1 variants with other psychiatric disorders, endophenotypes, and neurophysiological traits ${ }^{[6-68]}$. Rare missense mutations in DISC1 have been identified in patients with SCZ, BD, and MDD. In a previous study, a targeted resequencing was used to sequence the DISC1 locus $(528 \mathrm{~Kb})$ in 1,542 samples ${ }^{[69]}$. Two-thousand and ten rare variants $(\mathrm{MAF}<1 \%$ ) were found, and 489 variants were located in regulatory regions while 36 mapped to coding exons ${ }^{[69]}$. One variant, rs16856199, in DISC1 was found to be significant in a region-wide association test and burden analysis revealed an excess of rare regulatory variants in MDD patients. In addition, a rare missense mutation R37W in an MDD patient was found to be transmitted to two affected offspring.

\section{DISC1 pathway}

DISC1 functions as a scaffold protein in a large pathway comprised of two networks of genes, termed the "DISC1 Interactome" and "DISC1 Regulome". The "Interactome" describes a set of proteins that interact directly with the DISC1 protein, whilst the "Regulome" designates a set of genes that are regulated by DISC1 protein. Both networks converge on pathways critical for various neurodevelopmental processes and therapeutic targets of of SCZ, BD, and MDD. Genetic analyses of patients with psychiatric disorders have identified additional mutations in DISC1 pathway genes. For example, the GWAS studies have found common variants in SCZ candidate genes like, PDE4B, and sequencing of DISC1 and genes within the DISC1 pathway has revealed an excess of rare missense variants in SCZ cases versus controls ${ }^{[0,71]}$. Variations within the DISC1 pathway can have numerous effects on cognition as well as neurodevelopmental processes and signaling. Perturbations in the DISC1 pathway could represent an important susceptibility pathway in the development of SCZ.

In a recent study, a targeted resequencing analysis of 59 "DISC1 Interactome" and 154 "DISC1 Regulome" genes was performed in 654 cases with SCZ, BD or MDD, and in 889 healthy controls ${ }^{[72]}$. Burden analysis showed the gene translin-associated factor X-interacting protein 1 (TSNAXIP1) was enriched for rare damaging mutations in SCZ and MDD cases relative to controls. SKAT analysis showed an increased burden of singleton disruptive variants in the "DISC1 Regulome" in SCZ patients. There are significant associations between rare functional variants in the interactome with measures of cognitive performance. DISC1, MAP1A, CIT, and DST were found to be associated with cognitive ability based on burden analysis. Taken together these data suggest that genes in the "Regulome" and "Interactome" play a role in the etiology, progression, and severity of symptoms in $\mathrm{SCZ}^{[72]}$.

Targeted resequencing has been applied to sequence DISC1 and its interactors for SCZ and other neurodevelopmental disorders in other studies. Kenny et al. ${ }^{[71]}$ sequenced patients with ASD and SCZ in 215 synaptic genes including DISC1 Interactome genes known to be important to synaptic function. A significant burden of loss of function variants were found among combined cases of ASD and SCZ patients, which demonstrates that dysregulation at the synapse is an important feature of ASD and SCZ ${ }^{[71]}$. Moens et al. ${ }^{[70]}$ investigated rare coding variants in 10 DISC1 Interactome genes in 486 SCZ patients and 514 healthy controls from a Northern Swedish population. They found that rare mutations with MAF of 0.01 or less were found more often in SCZ cases than in controls. Unrelated studies of drug dependence have also 
demonstrated the significance of DISC1 pathway genes within the regulome as well, showing for instance, significant associations between rare variations found in DISC1 and GRIN2B and opioid dependence ${ }^{[73]}$.

\section{Other candidate genes and pathways}

Several other candidate genes have been implicated in SCZ, with a wide variety of functions. Rare variations in nuclear distribution $\mathrm{E}$ homolog 1 ( $N D E 1)$, a gene that plays a role in microtubule formation, mitosis, and neuronal migration ${ }^{[74]}$. One investigation identified variant $\mathrm{S} 214 \mathrm{~F}$, which was significantly associated with SCZ based on Fischer's exact test. This variant is deleterious to the function of the NDE1 protein ${ }^{[7]}$. The targeted sequencing of Northern Swedish SCZ patients revealed that NDE1 is significantly associated with SCZ diagnosis ${ }^{[70]}$. Dysfunction in myelination of axons, has also been implicated in SCZ patients. A rare variant in a receptor subunit for a myelin-associated inhibitor known as RTN46 was shown to have a modest association with $\mathrm{SCZ}^{[75]}$. This subunit has been demonstrated to play a role in synapse formation and is located on chromosome 22 in a mutational hotspot associated with SCZ. Upon further investigation, this variant was found to affect synaptogenesis by decreasing growth cone collapse ${ }^{[75]}$. Imputation analysis of GWAS data found both common and rare variants in ADAMTSL3 gene, a constituent of the extracellular matrix ${ }^{[76]}$. An additional investigation found four rare variants located in noncoding regions near the MIR185 gene, which codes for a microRNA with many functions ${ }^{[77]}$.

There is an overlap in many of the genes found to contain rare variation in SCZ with other neuropsychiatric and neurodevelopmental disorders like ASD. GRIN2B is a gene that is regulated by the DISC1 protein, implicating dysfunction in glutamateric signaling in SCZ neuropathology. In a study on ASD and SCZ, the investigators found an excess of rare variants, over 200 non-synonymous variants. Many of these rare variants had deleterious effects on genes associated with neurite outgrowth, Rett syndrome, and intellectual deficits ${ }^{[78]}$. Further investigations of the genetic overlap between SCZ and ASD have also demonstrated that the rate of de novo mutations in ASD spectrum disorder and SCZ patients was higher than that of controls ${ }^{[79]}$.

\section{WES FOR CODING VARIANTS}

WES is a method of sequencing that focuses on coding regions of the genome. It is cheaper than WGS, making it possible to sequence more samples, while potentially increasing the signal to noise ratio. However, the power of a WES study will depend on the number of samples and the amount of variation in the genes of interest ${ }^{[39]}$. By excluding de novo mutations and filtering of these variants in GWAS or WES can help to further narrow down the search for rare causative variants or as a means of grouping variants for association analyses ${ }^{[80]}$. Here, we review the WES studies for investigating rare coding variants associated with SCZ [Table 2].

\section{Case-control WES studies}

WES has been widely used in case-control studies to search for candidate genes associated SCZ. In a WES study of 4,264 SCZ cases and 9,343 controls, investigators found a significant association between loss of function mutations in SETDIA and SCZ. SETDIA is a gene that is associated with developmental disorders, which is further underscored by the fact that loss of function mutations in this gene are rare within the general population ${ }^{[81]}$. Damaging and disruptive ultra-rare mutations were found to be enriched in cases of SCZ. Investigators observed de novo nonsynonymous mutations in two genes previously identified as SCZ candidate genes, TAF13 and SETD1A. Additionally, A single ultra-rare variant in NRXN1 was identified to be associated with $\mathrm{SCZ}^{[82]}$. Giacopuzzi et al. ${ }^{[83]}$ sequenced an Italian cohort of 180 persons diagnosed with SCZ and detected 45 brain-expressed genes with deleterious effects. Four variants in four candidate genes ( $A N O 2$, FMN1, MEGF8 and GAD1) were determined to have rare or novel missense mutations which were not detected in control patients. The results indicate that high levels of homozygosity are associated with increased rare recessive variations in genes that convey a higher risk of $\mathrm{SCZ}^{[83]}$. WES studies of common and rare variants in SCZ risk loci can be used to elucidate psychiatric drug targets and improve treatment 
Table 2. Rare variants association studies

\begin{tabular}{|c|c|c|c|}
\hline Design & Samples & Risk genes & Authors \\
\hline \multicolumn{4}{|c|}{ Target resequencing } \\
\hline Case-control & $\begin{array}{l}240 \text { cases of SCZ, } 221 \text { cases of BD, and } 192 \text { cases } \\
\text { of MDD and } 889 \text { controls }\end{array}$ & DISC1 & Thomson et al. ${ }^{[69]}$ \\
\hline Case-control & $80 \mathrm{SCZ}$ cases and 80 controls & DISC1, ATF5, GRB2, YWHAE, ZNF365 & Moens et al. ${ }^{[70]}$ \\
\hline Case-control & 273 SCZ cases and 287 controls & $N R X N, N R L N, G R / N 2 B$ & Kenny et $a .^{[71]}$ \\
\hline Case-control & $\begin{array}{l}654 \text { cases ( } 241 \mathrm{SCZ}, 221 \mathrm{BD} \text { and } 192 \mathrm{MDD}) \text { and } \\
889 \text { controls }\end{array}$ & DISC1, TSNAXIP1 & Teng et $a l_{.}^{[72]}$ \\
\hline Case-control & $\begin{array}{l}760 \text { cases with co-occurring alcohol dependence, } \\
\text { cocaine dependence and opioid dependence, and } \\
760 \text { controls }\end{array}$ & $D / S C 1, G R / N 2 B$ & Xie et $a l^{[73]}$ \\
\hline Case-Control & $\begin{array}{l}433 \text { SCZ, } 145 \text { pervasive developmental disorders, } \\
3554 \text { controls }\end{array}$ & NDE1 & Kimura et al..$^{[74]}$ \\
\hline Case-control & $\begin{array}{l}\text { Set 1:370 SCZ, } 192 \text { ASD \& Set 2: } 1716 \text { SCZ, } 382 \\
\text { ASD, } 4009 \text { controls }\end{array}$ & RTN4R & Kimura et al. ${ }^{[75]}$ \\
\hline Case-control & 92 SCZ patients & ADAMTSL3 & Dow et al. ${ }^{[76]}$ \\
\hline Case-control & $\begin{array}{l}1000 \text { SCZ cases and } 500 \text { controls. Follow-up } \\
\text { analysis: } 3598 \text { and } 4082 \text { controls }\end{array}$ & ATAT1, SH3PXD2A, NTRK3, MIR185 & Forstner et al..$^{[77]}$ \\
\hline Case-control & $\begin{array}{l}142 \text { ASD patients, } 143 \text { SCZ subjects, and } 277 \\
\text { controls }\end{array}$ & ILIRAPLI, MAOB & Piton et $a l^{[78]}$ \\
\hline Case-control & 285 SCZ cases and 285 controls & SHANK3, IL1RAPL1, NRXN1 & Awadalla et $a{ }^{[79]}$ \\
\hline \multicolumn{4}{|l|}{ WES } \\
\hline Mixed & $\begin{array}{l}4,264 \text { SCZ cases, } 9,343 \text { controls and } 1,077 \\
\text { proband trios }\end{array}$ & SETD1A & Singh et $a{ }^{[81]}$ \\
\hline Case-control & $\begin{array}{l}12,332 \text { individuals ( } 4,877 \text { SCZ patients, } 6,242 \\
\text { controls and 1,144 other disorders) }\end{array}$ & TAF13, SETD1A, NRXN1 & Genovese et $a l^{[82]}$ \\
\hline Case-control & $\begin{array}{l}50 \text { controls and } 50 \text { patients (from } 180 \text { SCZ } \\
\text { patients) }\end{array}$ & MEGF8, GAD1, FMN1, ANO2 & Giacopuzzi et al.. ${ }^{[83]}$ \\
\hline Case-control & 36,989 cases and 113,075 controls & PSD-95, ARC, NMDAR & Ruderfer et $a{ }^{[110]}$ \\
\hline Case-control & $\begin{array}{l}\text { 100,296 individuals (mixture of cases and } \\
\text { controls) }\end{array}$ & & Ganna et $a l_{.}^{[85]}$ \\
\hline Case-control & $2,545 \mathrm{SCZ}$ cases and 2,545 controls & NSD1, HELLS, PHF21A, PAWR & Curtis $^{[86]}$ \\
\hline Case-control & 2,536 patients and 2,543 controls & $A R C, F M R P, F M R 1$ & Purcell et al. ${ }^{[14]}$ \\
\hline Family & 623 family trios & $A R C, F M R P, N M D A R$ complex genes & Fromer et al. ${ }^{[87]}$ \\
\hline Meta analysis & $\begin{array}{l}\text { Rare coding variants in } 9,274 \text { controls and } 4,133 \\
\text { SCZ cases, de novo mutations in } 1,077 \text { family } \\
\text { trios, and copy number variants from 6,882 cases } \\
\text { and } 11,255 \text { controls }\end{array}$ & & Singh et $a{ }^{[88]}$ \\
\hline Case-control & 2,545 cases and 2,545 controls & $\begin{array}{l}\text { Selected genes implicated: PLN, SLC25A4, } \\
\text { NDP, ARL1 }\end{array}$ & Trakadis et al. ${ }^{[89]}$ \\
\hline Family & 53 family trios \& 22 unrelated controls & $\begin{array}{l}\text { Selected genes implicated: ESAM, LAMA2, } \\
\text { RBICC1, SPATA }\end{array}$ & Xu et al. ${ }^{[91]}$ \\
\hline Family & 14 proband trios & $\begin{array}{l}\text { Selected genes: ZNF565, NRIP1, CCDC137, } \\
\text { CHD4 }\end{array}$ & Girard et al. ${ }^{[92]}$ \\
\hline Mixed & 14 parent child trios, 48 unrelated SCZ cases & PTPRG, SLC39A13, and TGM5 & Kranz et al. ${ }^{[93]}$ \\
\hline Family & 10 related individuals (3 diagnosed with SCZ) & TIMP2 & John et al. ${ }^{[94]}$ \\
\hline Mixed & $\begin{array}{l}\text { Multi-member family, } 1000 \text { cases \& 1,050 } \\
\text { ethnically matched controls, and } 310 \text { sporadic } \\
\text { cases of Affrican American and Caucasian origin }\end{array}$ & TAAR1 & John et al. ${ }^{[95]}$ \\
\hline Mixed & $\begin{array}{l}\text { Multiplex family: } 6 \text { affected, } 8 \text { unaffected, } \\
1 \text { unknown, resequencing: } 15 \text { members of a } \\
\text { multiplex family and } 111 \text { affected offspring }\end{array}$ & UNC13B & Egawa et al. ${ }^{[96]}$ \\
\hline Mixed & $\begin{array}{l}3 \text { families with one affected patients and an } \\
\text { unaffected sibling, } 96 \text { SCZ patients, } 638 \text { SCZ } \\
\text { patients and } 675 \text { controls }\end{array}$ & PDCD11 & Hoya et al. ${ }^{[97]}$ \\
\hline Mixed & $\begin{array}{l}\text { Targeted sequencing of } 2 \text { cases of COS, WES } 17 \\
\text { proband trios with sporadic COS }\end{array}$ & ATP1A3 \& FXYD & Chaumette et al. ${ }^{[98]}$ \\
\hline Mixed & UK10K: 1,392 SCZ cases and 982 persons with & IGTB4 & O'Brien et al. ${ }^{[99]}$ \\
\hline
\end{tabular}




\begin{tabular}{|c|c|c|c|}
\hline Mixed & 161 affected patients and 69 controls & FMRP, VGCCS & Salvoro et al. ${ }^{[100]}$ \\
\hline Family & 5 large families with multiple affected patients & GRM5, PPEF2 \& LRPIB & Timms et al. ${ }^{[101]}$ \\
\hline \multicolumn{4}{|l|}{ WGS } \\
\hline Family & 5 families with 3 or more affected individuals & SHANK2, SMARCA1 & Homann et al. ${ }^{[102]}$ \\
\hline Family & $\begin{array}{l}\text { A large Chinese family with several affected } \\
\text { members }\end{array}$ & RELN2 & Zhou et al. ${ }^{[103]}$ \\
\hline Family & $\begin{array}{l}8 \text { families of monozygotic twins who were } \\
\text { discordant for SCZ }\end{array}$ & TTN, GCN1L1, GAD1, PLXNA2, RELN2, FEX1 & Tang et al. ${ }^{[104]}$ \\
\hline Family & $\begin{array}{l}\text { Two trios of unaffected patients with children } \\
\text { with 22q11.2DS }\end{array}$ & $C O M T \& P R O D H$ & Chung et $a l^{[105]}$ \\
\hline \multirow[t]{2}{*}{ Case-control } & 1,616 samples & $D G C R 8$ & Gur et $a .^{[106]}$ \\
\hline & 9 unrelated individuals with 22 q11.2 deletions & $D G C R 8$ & Merico et al. ${ }^{[107]}$ \\
\hline Case-control & 188 patient, 376 controls & HHAT & Betcheva et al. ${ }^{[27]}$ \\
\hline Family & 91 SCZ families & $\begin{array}{l}\text { Selected genes implicated: } A B C A 13, \\
\text { CNTNAP2, SHANK2, SHANK3, HHAT }\end{array}$ & Khan et al. ${ }^{[108]}$ \\
\hline Family & $\begin{array}{l}660 \text { proband trios with at least one suicide } \\
\text { attempt }\end{array}$ & NRXN1 \& VIPR2 & Sokolowski et al. ${ }^{[109]}$ \\
\hline Family & 46 proband trios & $\begin{array}{l}\text { CNTNAP2, MAGI1, TSPAN7, CAV1, CAV2, } \\
\text { MET, ZIC1 }\end{array}$ & Piluso et $a l^{[110]}$ \\
\hline
\end{tabular}

ASD: autism spectrum disorder; BD: bipolar disorder; MDD: major depressive disorder; SCZ: schizophrenia; COS: childhood-onset schizophrenia; WES: whole exome sequencing; WGS: whole genome sequencing

outcomes. One such investigation found 10 of 167 pharmacologically relevant gene sets were enriched for deleterious rare variants in SCZ patients. When the investigators examined the enrichment of common variants in pharmacologically relevant gene sets, they found that 35 of 167 gene sets were enriched among SCZ patients. Interestingly, they found that when analyzing both common and rare variations, two gene sets of pharmacologically relevant drugs were associated with greater risk of SCZ, drugs that are meant to treat amoebiasis and protozoal diseases, as well as antipsychotics. Further analysis revealed 4 target genes associated with treatment efficacy in SCZ patients ${ }^{[84]}$.

Rare variations in genes that are intolerant to protein truncation variants have also been associated with increased risk in several neuropsychiatric disorders including $\mathrm{SCZ}^{[85]}$. Several pathways involved in cell cycle arrest, heterocycle metabolic processes, and covalent chromatin modification, and histone modification are enriched for rare functional variants. The findings demonstrated a role for dysfunction or dysregulation of the cell cycle and histone modification in the pathology of $\mathrm{SCZ}^{[86]}$. Rare disruptive mutations associated with SCZ are likely to play a role as patients have higher rates of rare disruptive mutation than healthy controls. Further investigation of three sets of genes associated with the activity-regulated cytoskeletonassociated protein (ARC), postsynaptic density 95 protein, and calcium ion channels, revealed that the number rare disruptive variants among these gene sets were increased in SCZ. Many of the rare disruptive variants found were non-synonymous de novo mutations ${ }^{[17]}$. In a case-control investigation, the scientists found that mutational rates for the diagnosed patients were correlated with advanced parental age. However, $79 \%$ of all de novo mutations occurred on paternal chromosomes. Patient exomes showed an increased rate in non-synonymous de novo mutations in genes associated with SCZ and synaptic function. Upon further investigation, it was shown that the proteins resulting from these non-synonymous mutations exhibited greater number of protein-protein interactions with synaptic proteins than was expected. Genes involved in forming the N-methyl-D-aspartate receptor (NMDAR) and ARC complexes, which are involved in processes underlying synaptic plasticity, were particularly enriched in non-synonymous mutations ${ }^{[87]}$. Many of the variations associated with SCZ are found within genes that are intolerant of damaging mutations. In a study of 9,274 controls and 4,133 SCZ cases, the authors compared rates of copy number variants between cases and control ${ }^{[88]}$. Investigators also performed joint analysis to determine if there were associations between cases and controls with respect to combinations of SNVs, copy number variants, and de novo mutations. The authors determined that SCZ patients had a greater number of variations in genes that are intolerant of loss of function mutations. Burden analysis also demonstrated that loss of function mutations were associated 
with intellectual disability in SCZ patients. Variations in several genes implicated in ASD were found to overlap with those associated with SCZ. The supervised learning method, gradient boosted trees, was used to determine whether combinations of inherited and de novo variants could be used to predict the risk of SCZ in WES data from 2,545 patients with SCZ and 2,545 controls ${ }^{[89]}$. The investigators reported that the algorithm was $85 \%$ accurate in identifying patients diagnosed with SCZ. The results support the polygenic model of SCZ. The investigators further argue that the results support the "threshold hypothesis", whereby SCZ is mainly a result of the accumulation of inherited mutations from an unaffected parent in susceptibility genes beyond a threshold.

\section{Family WES studies}

Familial studies can be advantageous in studying highly heritable disorders where the loci associated with diseases have been identified ${ }^{[90]}$. This strategy is likely to increase the signal of rare causal variants which may otherwise be dampened by the presence of common variants in association studies or burden analyses ${ }^{[40,90]}$. A previous WES study of 53 family trios of the first episode sporadic patients diagnosed as SCZ showed that rare de novo variants were 10 times more likely contain non-synonymous mutations than those inherited from parents ${ }^{\left[{ }_{11}\right]}$. In another investigation of de novo mutations in 14 trios, the researchers found the ratio of nonsense to missense mutations was higher than expected based on previous studies ${ }^{[92]}$. They concluded that de novo mutations likely accounted for the missing heritability in SCZ. Family studies can also be used to help identify genetic variants associated with complex disease within the general population. Investigators found consensus in de novo variations in the PTPRG, SLC39A13, and TGM5 genes between in 5 proband trios and 12 out of 48 unrelated cases of SCZ ${ }^{[93]}$. Family cohort studies can aid the understanding of the heritability of SCZ within families. In a WES study of an Indian family, the investigators found the variants in TIMP2 and PIWIL3 genes are segregated with family members who had been diagnosed with $\mathrm{SCZ}^{[94]}$. Pharmacological targets can also be elucidated through family cohort studies. In an investigation of an affected Indian family, WES revealed a rare heterozygous missense mutation in the TAAR1 gene, present only in the affected family members. This mutation affects a disulfide bond critical for the $G$ protein-coupled receptor and may have relevance to the discovery of pharmacological interventions for $\mathrm{SCZ}^{[95]}$. A separate investigation of two cases and one control in a family identified four rare variations in $U N C 13 B$ were unique to affected individuals ${ }^{[96]}$. In a study of three families affected by SCZ, PDCD11 was found to have recurrent variations and three variants were determined to be deleterious ${ }^{[97]}$. ATP1A3 is associated with alternating hemiplegia of childhood and was previously demonstrated to be associated with ASD. Chaumette et al. ${ }^{[98]}$ used WES to determine whether rare variations in this gene are associated with risk of childhood-onset SCZ in an American cohort. They found three variants, two de novo and one a non-synonymous, in the ATP1A3 gene and three rare variants in an interacting gene FXYD1. These genes were associated with a greater risk of $\mathrm{SCZ}^{[98]}$. ITGB4 gene is involved in cellular interactions and is highly expressed in neural stem cells and precursors. A gene-wise weighted burden test conducted using Bulgarian proband trios failed to find any gene that achieved exome wide significance, however, ITGB4 was the most "highly ranked" based on its signed $\log P$-value ${ }^{[99]}$.

Family WES studies can be used to identify pathways associated with a greater risk for developing SCZ. A study of a familial cohort combined WES and identity by descent model to map alleles associated with greater risk of $\mathrm{SCZ}^{[100]}$. Non-synonymous variants that were associated with greater risk for SCZ and were also enriched in pathways involved in the extracellular matrix and the development of neuronal projections. Although, future investigations might consider whether this variation might contribute to certain cognitive or behavioral symptoms ${ }^{[96]}$. Timms et al. ${ }^{[101]}$ investigated rare variants associated with the NMDA receptors in 5 families whose pedigrees demonstrate several persons affected by SCZ. They found rare variations in 21 genes, two of which were found in GRM5, a metabotropic glutamate receptor. Both variants affect the extracellular domain. Rare variants were also determined to occur in the PPEF2 and $L R P 1 B$ genes as well ${ }^{[101]}$. 


\section{WGS FOR GENOMIC VARIANTS}

WGS can lead to the identification of associations between genomic variants and SCZ [Table 2]. It can be used to identify small variants, including SNV and small insertions and deletions (INDELs), in coding and noncoding regions. Since the entire genome is sequenced, WGS is also a comprehensive tool for detecting large structural variants such as CNVs.

\section{Small variants}

WGS has given investigators the ability to investigate the association between rare small variations and disease. One investigation found deleterious missense mutations in the SHANK2 and SMARCA1 genes which play a role in the post-synaptic density and neurogenesis ${ }^{[102]}$. An investigation of a large Chinese family with several members diagnosed with SCZ found that the genetic model of inheritance was autosomal dominant and one missense variant in RELN, a gene involved in neuronal migration, was significantly associated with $S C Z^{[103]}$. The missense variant in RELN was found to co-segregate with the disorder and to be disruptive to protein function WGS of monozygotic twins discordant for SCZ indicates multiple genetic risk factors for $\mathrm{SCZ}^{[104]}$. The rate of de novo mutations was correlated with paternal age at the time of conception. A combination of inherited and de novo rare mutations seems to confer an increased risk of developing SCZ.

\section{Structural variants}

The 22q11.2 deletion syndrome is a common genetic disorder, which is associated with a 25 -fold increase in the risk of psychotic disorders, like $S C Z^{[105]}$. In order to study the effects of 22q11.2 deletions on phenotype and develop better diagnostic assessments, the International Brain and Behavior Consortium developed a framework for investigations of the genetics of this disorder using data from patients and unaffected adults $^{[106]}$. The model assumes that remaining haploid copies of segments of 22 q11.2 deletion sites will demonstrate an increased number of variants in individuals affected by SCZ or psychotic disorders. The combined model also tests for SNPs, for and rare structural variants, which exist outside of deleted 22q11.2 regions. The findings showed that patients with SCZ had significantly more CNVs than undiagnosed individuals $^{[106]}$. 22q11.2 deletions alone cannot explain the risk of developing SCZ. Investigators performed WGS in a group of nine unrelated patients, and demonstrated a greater burden for rare variants in cases than in controls in neurofunctional gene sets ${ }^{[107]}$. Burden analysis also revealed a large association between 22q11.2 microdeletions and rare variations in non-coding RNA genes as well. A familial study detected associations between variations in COMT and $P R O D H$ with cognitive impairments. Both the COMT and $P R O D H$ genes are located in the 22q11 deletion region ${ }^{[105]}$. Merico et al. ${ }^{[107]}$ performed WGS on 9 unrelated Canadian patients with SCZ to investigate the impact of 22q11.2 deletions on gene expression. The investigators identified rare variants in coding regions that were predicted to have damaging effects under a haploinsufficiency model. They found that the patients with SCZ had more deleterious rare variations in neuronal genes associated with neural projection as well as neuronal function. The investigators then investigated the association between SCZ and rare variants in genes that are affected by DGCR8, a gene involved in miRNA biogenesis. They found that patients with 22q11.2 deletions, where the function of DGCR8 was affected, were at greater risk of developing SCZ. These results lend further credence to the miRNA hypothesis of $S C Z^{[107]}$.

WGS was also applied to family studies to discover variants or modifications that could explain the variability of SCZ phenotypes. Khan et al. ${ }^{[108]}$ performed WGS to search for CNVs in 91 families with members diagnosed as schizophrenic. They found that there was an average of 9 rare CNVs per patient. Intronic deletions were found to be 3 times greater than exonic deletions. The transmission rates for rare exonic CNVs from unaffected parents to affected children were higher than for unaffected children ${ }^{[108]}$. A WGS study on 660 proband trios found rare CNVs in 14 loci that overlapped with SCZ risk genes in nine children seven offspring of persons who had attempted suicide. Forty-five of the patients that previously attempted suicide were found to have CNVs in 65 genes known to be involved in neural development ${ }^{[109]}$. 
Piluso et al. ${ }^{[110]}$ investigated de novo CNVs in cis-regulatory elements using NGS data from 46 family trios where at least one member was diagnosed with SCZ. They found de novo CNVs in genes associated with SCZ, intellectual disability, neuronal, migration, and neural development: CNTNAP2, MAGI1, TSPAN7, $C A V 1, C A V 2, M E T$, and ZIC1. Two de novo mutations were found to affect the hs1043 and hs582 regulatory elements $^{[110]}$.

\section{CHALLENGES}

One challenge of investigating the genetics of SCZ is that many of the variants, which seem to confer susceptibility to SCZ or to have demonstrated an association with SCZ overlap with other neuropsychiatric illnesses including BD and MDD. In an investigation of over 19,000 cases of SCZ and BD, the investigators identified 219 SNPs that achieved genome-wide significance. These variants were found within 6 regions of the genome, two which were previously associated with SCZ, MHC, MAD1L1; two previously associated with BD, TRANK1, IFI44L, one gene that was based on previous work was associated with both diseases, CACNA1C; and a novel risk gene, PIK3C2A, also associated with both SCZ and BD. None of the 6 genes identified demonstrated a significant difference in terms of odds ratio between SCZ or BD, which further demonstrates the genetic overlap between the polygenic nature of these disorders ${ }^{[111]}$. The complex genetics of SCZ has also been demonstrated by the fact that it is difficult to distinguish between SCZ, schizoaffective, schizotypal, and non-affective psychotic disorders, which is sometimes referred to as the SCZ spectrum. In an investigation of the polygenic nature of SCZ spectrum disorders, the investigators calculated polygenetic risk scores for each subject based on the genome-wide significance of SNP in cases vs. controls. Individuals who lacked a psychiatric diagnosis had significantly lower scores than those of cases who were diagnosed on the spectrum ${ }^{[112]}$. SCZ and ASD also share a number of similar endophenotypes with respect to executive function, cognition, and sensory deficits ${ }^{[113]}$. Alterations in the morphology and neurochemistry of several neuroanatomical regions are common to both SCZ and ASD, including the prefrontal cortex, anterior cingulate, posterior cingulate, claustrum, and right parahippocampal gyrus among others ${ }^{[114-116]}$. In terms of genetic overlap, studies have found increased number of de novo splice site mutations and CNVs associated with both SCZ and ASD ${ }^{[117,118]}$. The common susceptibility genes associated with SCZ and ASD are post synaptic density associated genes like $D L G 2, N R X N 1$, and $B D N F^{[119-121]}$. Factors relating to prenatal development represent another point similarity in the etiology of ASD and SCZ, particularly with respect to inflammation and neuroinflammation ${ }^{[121,122]}$. It is also worth noting that maternal infection and autoimmune disorders are associated with greater risk of developing ASD and $\mathrm{SCZ}^{[1,123]}$.

Another challenge is determining the role of environmental factors. The rate of the immune system's exposure to bacteria is higher early in life and decreases with age. Impaired immune response in persons has been hypothesized to play a role in the development of SCZ ${ }^{[113,124]}$. However, most studies implicate maternal infection as a greater risk factor than postnatal immune insults ${ }^{[14,125]}$. Inferior social status is an environmental factor that may also play role in the increased risk of SCZ diagnoses ${ }^{[108]}$. These differences in environmental conditions between racial and ethnic minorities may result in neurobiological changes as well. In two Canadian investigations of clients at risk for psychosis and SCZ and healthy controls, researchers found that immigrants demonstrated increased dopamine release from the striatum relative to non-immigrants ${ }^{[126,127]}$. However, the mean genetic variability did not differ among the ethnic groups ${ }^{[128]}$. The racial or ethnic differences in the incidence of SCZ diagnosis are studies, which have shown that socioeconomic status and perceived honesty of the patient may influence diagnoses. In one investigation interviewers diagnosed $19 \%$ of white participants as schizophrenic, however study interviewers diagnosed nearly half of the black participants ${ }^{[129]}$. Socioeconomic factors, age, substance use, and reports of symptoms such as hallucinations differed between the two groups. However, investigators found that the client's race still increased the likelihood of SCZ diagnosis three-fold ${ }^{[129]}$. A literature review concerning the black Caribbean populations in the UK found several possible explanations for the increased incidence of SCZ in 
these populations relative to white British persons ${ }^{[130]}$. The data suggests a genetic component as the risk of SCZ increases with the relatedness of an individual to persons affected by SCZ. The risk of developing SCZ increases the most for individuals with affected members of their immediate family, from $6 \%-13 \%$. Other possible explanations include a predisposition for migration in schizophrenic individuals, misdiagnosis, drug use, and various socioeconomic factors. In a retrospective cohort study, investigators examined racial/ ethnic disparities in SCZ diagnoses. The authors examined three classes of factors related to SCZ diagnoses, predisposing factors, demographic measures like age and ethnicity; enabling factors, such as insurance status and socioeconomic measures; and need factors, such as symptoms and substance use. The investigators found that the predisposing factors of male gender, Hispanic ethnicity, and African American race increased the likelihood of SCZ diagnosis ${ }^{[131]}$. A study with found that while polygenic risk scores were efficacious in distinguishing schizophrenic patients from healthy controls of European ancestry, that polygenic risk scores were less efficacious with African American participants ${ }^{[132]}$.

Detection of transcriptional signatures of SCZ provides new insight into the genetics basis of the diseases. To study the role of the microbiome in the etiology of SCZ, Olde Loohuis et al ${ }^{[133]}$, collected the blood of 192 participants including patients with SCZ and BD and performed RNAseq to determine which microbes were present. Patients diagnosed with SCZ were found to have a more diverse microbiome than the other participant groups. After comparing SCZ cases to controls, the investigators determined that this was due to an overall increase in the microbial burden among SCZ patients ${ }^{[133]}$. A transcriptional analysis of lymphoblastoid cell lines from 268 cases and 446 controls showed 1,058 genes that were differentially expressed between SCZ cases and healthy controls ${ }^{[134]}$. A further analysis of the gene expression data found that SCZ cases demonstrated upregulation of genes related to immunological function and downregulation of genes related to apoptosis or non-immune functions ${ }^{[134]}$. Schizophrenics were also demonstrated to have a greater number of copies of the ribosomal RNA gene than healthy controls ${ }^{[135]}$. Brennand et al. ${ }^{[136]}$ used human induced pluripotent stem cells (hIPSC) derived neurons to model the neurobiology underlying SCZ. They found reduced arborization and synaptic contacts in the hIPSC neurons of SCZ patients, though spontaneous neuronal activity was found to be normal. They found 596 genes that were differentially expressed between the hIPSC neurons of SCZ patients and those of healthy controls. Treatment of hIPSC neurons with loxapine, an antipsychotic, increased the number of synaptic contacts and the expression of receptors crucial in glutamatergic signaling ${ }^{[136]}$.

\section{CONCLUSION}

SCZ is a serious mental disorder affecting millions of people worldwide, but the underlying mechanisms remain unknown. The common variants identified by the GWAS only explain a small fraction of the estimated SCZ heritability. The development of NGS provides an unprecedented opportunity to identify the SCZ candidate genes and variants and research their functions. The analysis of the rare variants is timely and achievable in its aims of understanding the relationship between genotype and phenotype underlying SCZ. Multiple bioinformatics tools have been developed to detect rare variant associations in case-control and family studies. Targeted resequencing, WES and WGS have been used to identify the rare variants associated with SCZ. Although there are numerous challenges in SCZ research, the rare variant studies can provide useful information for characterizing the rare mutations and elucidating the genetic mechanisms by which the variations cause the SCZ and other mental illnesses. It could help biomedical scientists develop better diagnostic and treatments for individuals with psychiatric disorders.

\section{DECLARATIONS}

\section{Acknowledgments}

We thank supports from the Howard University Junior Faculty Writing \& Creative Works Summer Academy. 


\section{Authors' contributions}

Idea conception, literature research and manuscript revision: Rhoades R, Jackson F, Teng S

Manuscript writing and editing: Rhoades R, Teng S

\section{Availability of data and materials}

Not applicable.

\section{Financial support and sponsorship}

This work was supported by the Howard University startup funds (U100193). It was supported (in part) by the National Institute on Minority Health and Health Disparities of the National Institutes of Health under Award Number G12MD007597. The content is solely the responsibility of the authors and does not necessarily represent the official views of the National Institutes of Health.

\section{Conflicts of interest}

All authors declared that there are no conflicts of interest.

\section{Ethical approval and consent to participate}

Not applicable.

\section{Consent for publication}

Not applicable.

\section{Copyright}

(c) The Author(s) 2019.

\section{REFERENCES}

1. Wu EQ, Shi L, Birnbaum H, Hudson T, Kessler R. Annual prevalence of diagnosed schizophrenia in the USA: a claims data analysis approach. Psychol Med 2006;36:1535-40.

2. Wu EQ, Birnbaum HG, Shi L, Ball DE, Kessler RC, et al. The economic burden of schizophrenia in the United States in 2002. J Clin Psychiatry 2005;66:1122-9.

3. Cloutier M, Aigbogun MS, Guerin A, Nitulescu R, Ramanakumar AV, et al. The economic burden of schizophrenia in the United States in 2013. J Clin Psychiatry 2016;77:764-71.

4. Schultz SH, North SW, Shields CG. Schizophrenia: a review. Am Fam Physician 2007;75:1821-9.

5. Millier A, Schmidt U, Angermeyer MC, Chauhan D, Murthy V, et al. Humanistic burden in schizophrenia: a literature review. J Psychiatr Res 2014;54:85-93.

6. Marsman A, van den Heuvel MP, Klomp DW, Kahn RS, Luijten PR, et al. Glutamate in schizophrenia: a focused review and meta-analysis of 1H-MRS studies. Schizophr Bull 2013;39:120-9.

7. Plitman E, Nakajima S, de la Fuente-Sandoval C, Gerretsen P, Chakravarty MM, et al. Glutamate-mediated excitotoxicity in schizophrenia: a review. Eur Neuropsychopharmacol 2014;24:1591-605.

8. Egerton A, Modinos G, Ferrera D, McGuire P. Neuroimaging studies of GABA in schizophrenia: a systematic review with meta-analysis. Transl Psychiatry 2017;7:e1147.

9. Van Den Heuvel MP, Fornito A. Brain networks in schizophrenia. Neuropsychol Rev 2014;24:32-48.

10. Morgan C, Fisher H. Environment and schizophrenia: environmental factors in schizophrenia: childhood trauma - a critical review. Schizophr Bull 2007;33:3-10.

11. Cannon TD, Kaprio J, Lönnqvist J, Huttunen M, Koskenvuo M. The genetic epidemiology of schizophrenia in a Finnish twin cohort: a population-based modeling study. Arch Gen Psychiatry 1998;55:67-74.

12. Keller MC, Miller G. Resolving the paradox of common, harmful, heritable mental disorders: which evolutionary genetic models work best? Behav Brain Sci 2006;29:385-404.

13. Kim Y, Zerwas S, Trace SE, Sullivan PF. Schizophrenia genetics: where next? Schizophr Bull 2011;37:456-63.

14. International Schizophrenia Consortium, Purcell SM, Wray NR, Stone JL, Visscher PM, et al. Common polygenic variation contributes to risk of schizophrenia and bipolar disorder. Nature 2009;460:748-52.

15. Chan MK, Cooper JD, Heilmann-Heimbach S, Frank J, Witt SH, et al. Associations between SNPs and immune-related circulating proteins in schizophrenia. Sci Rep 2017;7:12586

16. Hawi Z, Tong J, Dark C, Yates H, Johnson B, et al. The role of cadherin genes in five major psychiatric disorders: a literature update. Am J 
Med Genet B Neuropsychiatr Genet 2018;177:168-80.

17. Morris JA. The genomic load of deleterious mutations: relevance to death in infancy and childhood. Front Immunol 2015;6:105.

18. Purcell SM, Moran JL, Fromer M, Ruderfer D, Solovieff N, et al. A polygenic burden of rare disruptive mutations in schizophrenia. Nature 2014;506:185-90.

19. Schork NJ, Murray SS, Frazer KA, Topol EJ. Common vs. rare allele hypotheses for complex diseases. Curr Opin Genet Dev 2009; 19:212-9.

20. Van Dongen J, Boomsma DI. The evolutionary paradox and the missing heritability of schizophrenia. Am J Med Genet B Neuropsychiatr Genet 2013;162B:122-36.

21. Ripke S, Neale BM, Corvin A, Walters JT, Farh KH, et al. Biological insights from 108 schizophrenia-associated genetic loci. Nature 2014;511:421-7.

22. Gibson G. Rare and common variants: twenty arguments. Nat Rev Genet 2012;13:135-45.

23. Hirschhorn JN, Daly MJ. Genome-wide association studies for common diseases and complex traits. Nat Rev Genet 2005;6:95-108.

24. Visscher PM, Brown MA, McCarthy MI, Yang J. Five years of GWAS discovery. Am J Hum Genet 2012;90:7-24.

25. Stranger BE, Stahl EA, Raj T. Progress and promise of genome-wide association studies for human complex trait genetics. Genetics 2011;187:367-83.

26. Barrett JC, Hansoul S, Nicolae DL, Cho JH, Duerr RH, et al. Genome-wide association defines more than 30 distinct susceptibility loci for Crohn's disease. Nat Genet 2008;40:955-62.

27. Betcheva ET, Yosifova AG, Mushiroda T, Kubo M, Takahashi A, et al. Whole-genome-wide association study in the Bulgarian population reveals HHAT as schizophrenia susceptibility gene. Psychiatr Genet 2013;23:11-9.

28. Ren HY, Wang Q, Lei W, Zhang CC, Li YF, et al. The common variants implicated in microstructural abnormality of first episode and drugnaïve patients with schizophrenia. Sci Rep 2017;7:11750.

29. Ripke S, Sanders AR, Kendler KS, Levinson DF, Sklar P, et al. Genome-wide association study identifies five new schizophrenia loci. Nat Genet 2011;43:969-76.

30. Mahmoudi E, Cairns MJ. MiR-137: an important player in neural development and neoplastic transformation. Mol Psychiatry 2017;22:44-55.

31. Liu C, Bousman CA, Pantelis C, Skafidas E, Zhang D, et al. Pathway-wide association study identifies five shared pathways associated with schizophrenia in three ancestral distinct populations. Transl Psychiatry 2017;7:e1037.

32. Schaid DJ, Chen W, Larson NB. From genome-wide associations to candidate causal variants by statistical fine-mapping. Nat Rev Genet 2018;19:491-504.

33. Mitchell KJ, Porteous DJ. Rethinking the genetic architecture of schizophrenia. Psychol Med 2011;41:19-32.

34. Farrell MS, Werge T, Sklar P, Owen MJ, Ophoff RA, et al. Evaluating historical candidate genes for schizophrenia. Mol Psychiatry $2015 ; 20: 555-62$.

35. Klein C, Lohmann K, Ziegler A. The promise and limitations of genome-wide association studies. JAMA 2012;308:1867-8.

36. Altmüller J, Budde BS, Nürnberg P. Enrichment of target sequences for next-generation sequencing applications in research and diagnostics. Biol Chem 2014;395:231-7.

37. Seleman M, Hoyos-Bachiloglu R, Geha RS, Chou J. Uses of next-generation sequencing technologies for the diagnosis of primary immunodeficiencies. Front Immunol 2017;8:847.

38. Salk JJ, Schmitt MW, Loeb LA. Enhancing the accuracy of next-generation sequencing for detecting rare and subclonal mutations. Nat Rev Genet 2018;19:269-85.

39. Kiezun A, Garimella K, Do R, Stitziel NO, Neale BM, et al. Exome sequencing and the genetic basis of complex traits. Nat Genet 2012;44:623-30.

40. Lee S, Abecasis GR, Boehnke M, Lin X. Rare-variant association analysis: study designs and statistical tests. Am J Hum Genet 2014;95:5-23.

41. Auer PL, Lettre G. Rare variant association studies: considerations, challenges and opportunities. Genome Med 2015;7:16.

42. Purcell S, Neale B, Todd-Brown K, et al. PLINK: a tool set for whole-genome association and population-based linkage analyses. Am J Hum Genet 2007;81:559-75.

43. Verma SS, Ritchie MD. Another round of "clue" to uncover the mystery of complex traits. Genes (Basel) 2018; doi: 10.3390/genes9020061.

44. Kao PY, Leung KH, Chan LW, Yip SP, Yap MK. Pathway analysis of complex diseases for GWAS, extending to consider rare variants, multi-omics and interactions. Biochim Biophys Acta Gen Subj 2017;1861:335-53.

45. Kaakinen M, Mägi R, Fischer K, Heikkinen J, Järvelin MR, et al. MARV: a tool for genome-wide multi-phenotype analysis of rare variants. BMC Bioinformatics 2017;18:110.

46. Morgenthaler S, Thilly WG. A strategy to discover genes that carry multi-allelic or mono-allelic risk for common diseases: a cohort allelic sums test (CAST). Mutat Res 2007;615:28-56.

47. Li B, Leal SM. Methods for detecting associations with rare variants for common diseases: application to analysis of sequence data. Am J Hum Genet 2008;83:311-21.

48. Neale BM, Rivas MA, Voight BF, Altshuler D, Devlin B, et al. Testing for an unusual distribution of rare variants. PLoS Genet 2011; doi: 10.1371/journal.pgen.1001322.

49. Wu MC, Lee S, Cai T, Li Y, Boehnke M, et al. Rare-variant association testing for sequencing data with the sequence kernel association test. Am J Hum Genet 2011;89:82-93.

50. Lee S, Emond MJ, Bamshad MJ, Barnes KC, Rieder MJ, et al. Optimal unified approach for rare-variant association testing with application 
to small-sample case-control whole-exome sequencing studies. Am J Hum Genet 2012;91:224-37.

51. Hasegawa T, Kojima K, Kawai Y, Misawa K, Mimori T, et al. AP-SKAT: highly-efficient genome-wide rare variant association test. BMC Genomics 2016;17:745.

52. Zhou JJ, Hu T, Qiao D, Cho MH, Zhou H. Boosting gene mapping power and efficiency with efficient exact variance component tests of single nucleotide polymorphism sets. Genetics 2016;204:921-31.

53. Chiu CY, Jung J, Wang Y, Weeks DE, Wilson AF, et al. A comparison study of multivariate fixed models and gene association with multiple traits (GAMuT) for next-generation sequencing. Genet Epidemiol 2017;41:18-34.

54. Chung RH, Tsai WY, Kang CY, Yao PJ, Tsai HJ, et al. FamPipe: an automatic analysis pipeline for analyzing sequencing data in families for disease studies. PLoS Comput Biol 2016; doi: 10.1371/journal.pcbi.1004980.

55. Jiang D, McPeek MS. Robust rare variant association testing for quantitative traits in samples with related individuals. Genet Epidemiol 2014;38:10-20.

56. Chen H, Meigs JB, Dupuis J. Sequence kernel association test for quantitative traits in family samples. Genet Epidemiol 2013;37:196-204.

57. Hu H, Roach JC, Coon H, Guthery SL, Voelkerding KV, et al. A unified test of linkage analysis and rare-variant association for analysis of pedigree sequence data. Nat Biotechnol 2014;32:663-9.

58. Wang X, Lee S, Zhu X, Redline S, Lin X. GEE-based SNP set association test for continuous and discrete traits in family-based association studies. Genet Epidemiol 2013;37:778-86.

59. Chen MH, Yang Q. RVFam: an R package for rare variant association analysis with family data. Bioinformatics 2016;32:624-6.

60. Wang X, Zhao X, Zhou J. Testing rare variants for hypertension using family-based tests with different weighting schemes. BMC Proc 2016:10:233-7.

61. Choi S, Lee S, Cichon S, Nöthen MM, Lange C, et al. FARVAT: a family-based rare variant association test. Bioinformatics 2014;30:3197205.

62. Werling DM, Brand H, An JY, Stone MR, Zhu L, et al. An analytical framework for whole-genome sequence association studies and its implications for autism spectrum disorder. Nat Genet 2018;50:727-36.

63. Natsoulis G, Bell JM, Xu H, Buenrostro JD, Ordonez H, et al. A flexible approach for highly multiplexed candidate gene targeted resequencing. PLoS One 2011; doi: 10.1371/journal.pone.0021088.

64. Edwards TL, Song Z, Li C. Enriching targeted sequencing experiments for rare disease alleles. Bioinformatics 2011;27:2112-8.

65. Blackwood DH, Fordyce A, Walker MT, St Clair DM, Porteous DJ, et al. Schizophrenia and affective disorders--cosegregation with a translocation at chromosome 1q42 that directly disrupts brain-expressed genes: clinical and P300 findings in a family. Am J Hum Genet 2001;69:428-33.

66. Carless MA, Glahn DC, Johnson MP, Curran JE, Bozaoglu K, et al. Impact of DISC1 variation on neuroanatomical and neurocognitive phenotypes. Mol Psychiatry 2011;16:1096-104.

67. Bradshaw NJ, Porteous DJ. DISC1-binding proteins in neural development, signalling and schizophrenia. Neuropharmacology 2012;62:1230-41.

68. Johnstone M, Thomson PA, Hall J, McIntosh AM, Lawrie SM, et al. DISC1 in schizophrenia: genetic mouse models and human genomic imaging. Schizophr Bull 2011;37:14-20.

69. Thomson PA, Parla JS, McRae AF, Kramer M, Ramakrishnan K, et al. 708 common and 2010 rare DISC1 locus variants identified in 1542 subjects: analysis for association with psychiatric disorder and cognitive traits. Mol Psychiatry 2014;19:668-75.

70. Moens LN, De Rijk P, Reumers J, Van den Bossche MJ, Glassee W, et al. Sequencing of DISC1 pathway genes reveals increased burden of rare missense variants in schizophrenia patients from a northern Swedish population. PLoS One 2011;6:e23450.

71. Kenny EM, Cormican P, Furlong S, Heron E, Kenny G, et al. Excess of rare novel loss-of-function variants in synaptic genes in schizophrenia and autism spectrum disorders. Mol Psychiatry 2014;19:872-9.

72. Teng S, Thomson PA, McCarthy S, Kramer M, Muller S, et al. Rare disruptive variants in the DISC1 interactome and regulome: association with cognitive ability and schizophrenia. Mol Psychiatry 2018;23:1270-7.

73. Xie P, Kranzler HR, Krystal JH, Farrer LA, Zhao H, et al. Deep resequencing of 17 glutamate system genes identifies rare variants in DISC1 and GRIN2B affecting risk of opioid dependence. Addict Biol 2014;19:955-64.

74. Kimura H, Tsuboi D, Wang C, Kushima I, Koide T, et al. Identification of rare, single-nucleotide mutations in NDE1 and their contributions to schizophrenia susceptibility. Schizophr Bull 2015;41:744-53.

75. Kimura H, Fujita Y, Kawabata T, Ishizuka K, Wang C, et al. A novel rare variant R292H in RTN4R affects growth cone formation and possibly contributes to schizophrenia susceptibility. Transl Psychiatry 2017; doi: 10.1038/tp.2017.170.

76. Dow DJ, Huxley-Jones J, Hall JM, Francks C, Maycox PR, et al. ADAMTSL3 as a candidate gene for schizophrenia: gene sequencing and ultra-high density association analysis by imputation. Schizophr Res 2011;127:28-34.

77. Forstner AJ, Basmanav FB, Mattheisen M, Böhmer AC, Hollegaard MV, et al. Investigation of the involvement of MIR185 and its target genes in the development of schizophrenia. J Psychiatry Neurosci 2014;39:386-96.

78. Piton A, Gauthier J, Hamdan FF, Lafrenière RG, Yang Y, et al. Systematic resequencing of X-chromosome synaptic genes in autism spectrum disorder and schizophrenia. Mol Psychiatry 2011;16:867-80.

79. Awadalla P, Gauthier J, Myers RA, Casals F, Hamdan FF, et al. Direct measure of the de novo mutation rate in autism and schizophrenia cohorts. Am J Hum Genet 2010;87:316-24.

80. Zuk O, Schaffner SF, Samocha K, Do R, Hechter E, et al. Searching for missing heritability: designing rare variant association studies. Proc Natl Acad Sci U S A 2014;111:E455-64.

81. Singh T, Kurki MI, Curtis D, Purcell SM, Crooks L, et al. Rare loss-of-function variants in SETD1A are associated with schizophrenia and 
developmental disorders. Nat Neurosci 2016;19:571-7.

82. Genovese G, Fromer M, Stahl EA, Ruderfer DM, Chambert K, et al. Increased burden of ultra-rare protein-altering variants among 4,877 individuals with schizophrenia. Nat Neurosci 2016;19:1433-41.

83. Giacopuzzi E, Gennarelli M, Minelli A, Gardella R, Valsecchi P, et al. Exome sequencing in schizophrenic patients with high levels of homozygosity identifies novel and extremely rare mutations in the GABA/glutamatergic pathways. PLoS One 2017;12:e0182778.

84. Ruderfer DM, Charney AW, Readhead B, Kidd BA, Kähler AK, et al. Polygenic overlap between schizophrenia risk and antipsychotic response: a genomic medicine approach. Lancet Psychiatry 2016;3:350-7.

85. Ganna A, Satterstrom FK, Zekavat SM, Das I, Kurki MI, et al. Quantifying the impact of rare and ultra-rare coding variation across the phenotypic spectrum. Am J Hum Genet 2018;102:1204-11.

86. Curtis D. Pathway analysis of whole exome sequence data provides further support for the involvement of histone modification in the aetiology of schizophrenia. Psychiatr Genet 2016;26:223-7.

87. Fromer M, Pocklington AJ, Kavanagh DH, Williams HJ, Dwyer S, et al. De novo mutations in schizophrenia implicate synaptic networks. Nature 2014;506:179-84.

88. Singh T, Walters JTR, Johnstone M, Curtis D, Suvisaari J, et al. The contribution of rare variants to risk of schizophrenia in individuals with and without intellectual disability. Nat Genet 2017;9:1167-73.

89. Trakadis YJ, Sardaar S, Chen A, Fulginiti V, Krishnan A. Machine learning in schizophrenia genomics, a case-control study using 5,090 exomes. Am J Med Genet Part B Neuropsychiatr Genet 2018; doi: 10.1002/ajmg.b.32638.

90. Bahlo M, Tankard R, Lukic V, Oliver KL, Smith KR. Using familial information for variant filtering in high-throughput sequencing studies. Hum Genet 2014;133:1331-41.

91. Xu B, Roos JL, Dexheimer P, Boone B, Plummer B, et al. Exome sequencing supports a de novo mutational paradigm for schizophrenia. Nat Genet 2011;43:864-8.

92. Girard SL, Gauthier J, Noreau A, Xiong L, Zhou S, et al. Increased exonic de novo mutation rate in individuals with schizophrenia. Nat Genet 2011;43:860-3.

93. Kranz TM, Harroch S, Manor O, Lichtenberg P, Friedlander Y, et al. De novo mutations from sporadic schizophrenia cases highlight important signaling genes in an independent sample. Schizophr Res 2015;166:119-24.

94. John J, Sharma A, Kukshal P, Bhatia T, Nimgaonkar VL, et al. Rare variants in tissue inhibitor of metalloproteinase 2 as a risk factor for schizophrenia: evidence from familial and cohort analysis. Schizophr Bull 2019;45:256-63

95. John J, Kukshal P, Bhatia T, Chowdari KV, Nimgaonkar VL, et al. Possible role of rare variants in trace amine associated receptor 1 in schizophrenia. Schizophr Res 2017;189:190-5.

96. Egawa J, Hoya S, Watanabe Y, Nunokawa A, Shibuya M, et al. Rare UNC13B variations and risk of schizophrenia: whole-exome sequencing in a multiplex family and follow-up resequencing and a case-control study. Am J Med Genet B Neuropsychiatr Genet 2016;171:797-805.

97. Hoya S, Watanabe Y, Hishimoto A, Nunokawa A, Kaneko N, et al. Rare PDCD11 variations are not associated with risk of schizophrenia in Japan. Psychiatry Clin Neurosci 2017;71:780-8.

98. Chaumette B, Ferrafiat V, Ambalavanan A, Goldenberg A, Dionne-Laporte A, et al. Missense variants in ATP1A3 and FXYD gene family are associated with childhood-onset schizophrenia. Mol Psychiatry 2018; doi: 10.1038/s41380-018-0103-8.

99. O’Brien NL, Fiorentino A, Curtis D, Rayner C, Petrosellini C, et al. Rare variant analysis in multiply affected families, association studies and functional analysis suggest a role for the ITGB4 gene in schizophrenia and bipolar disorder. Schizophrenia Research 2018;199:181-8.

100. Salvoro C, Bortoluzzi S, Coppe A, Valle G, Feltrin E, et al. Rare risk variants identification by identity-by-descent mapping and wholeexome sequencing implicates neuronal development pathways in schizophrenia and bipolar disorder. Mol Neurobiol 2018:55:7366-76.

101. Timms AE, Dorschner MO, Wechsler J, Choi KY, Kirkwood R, et al. Support for the N-methyl-d-aspartate receptor hypofunction hypothesis of schizophrenia from exome sequencing in multiplex families. JAMA Psychiatry 2013;70:582-90.

102. Homann OR, Misura K, Lamas E, Sandrock RW, Nelson P, et al. Whole-genome sequencing in multiplex families with psychoses reveals mutations in the SHANK2 and SMARCA1 genes segregating with illness. Mol Psychiatry 2016;21:1690-5.

103. Zhou Z, Hu Z, Zhang L, Hu Z, Liu H, et al. Identification of RELN variation p.Thr3192Ser in a Chinese family with schizophrenia. Sci Rep 2016;6:24327.

104. Tang J, Fan Y, Li H, Xiang Q, Zhang DF, et al. Whole-genome sequencing of monozygotic twins discordant for schizophrenia indicates multiple genetic risk factors for schizophrenia. J Genet Genomics 2017;20;44:295-306.

105. Chung JH, Cai J, Suskin BG, Zhang Z, Coleman K, et al. Whole-genome sequencing and integrative genomic analysis approach on two 22q11.2 deletion syndrome family trios for genotype to phenotype correlations. Hum Mutat 2015;36:797-807.

106. Gur RE, Bassett AS, McDonald-Mcginn DM, Bearden CE, Chow E, et al. A neurogenetic model for the study of schizophrenia spectrum disorders: the International 22q11.2 Deletion Syndrome Brain Behavior Consortium. Mol Psychiatry 2017;22:1664-72.

107. Merico D, Zarrei M, Costain G, Ogura L, Alipanahi B, et al. Whole-genome sequencing suggests schizophrenia risk mechanisms in humans with 22q11.2 deletion syndrome. G3 (Bethesda) 2015;5:2453-61.

108. Khan FF, Melton PE, McCarthy NS, Morar B, Blangero J, et al. Whole genome sequencing of 91 multiplex schizophrenia families reveals increased burden of rare, exonic copy number variation in schizophrenia probands and genetic heterogeneity. Schizophr Res 2018; doi: 10.1016/j.schres.2018.02.034.

109. Sokolowski M, Wasserman J, Wasserman D. Rare CNVs in suicide attempt include schizophrenia-associated loci and neurodevelopmental genes: a pilot genome-wide and family-based study. PLoS One 2016; doi: 10.1371/journal.pone.0168531.

110. Piluso G, Monteleone P, Galderisi S, Giugliano T, Bertolino A, et al. Assessment of de novo copy-number variations in Italian patients with 
schizophrenia: detection of putative mutations involving regulatory enhancer elements. World J Biol Psychiatry 2017;20:1-11.

111. Ruderfer DM, Fanous AH, Ripke S, McQuillin A, Amdur RL, et al. Polygenic dissection of diagnosis and clinical dimensions of bipolar disorder and schizophrenia. Mol Psychiatry 2014;19:1017-24.

112. Bigdeli TB, Bacanu SA, Webb BT, Walsh D, O’Neill FA, et al. Molecular validation of the schizophrenia spectrum. Schizophr Bull 2014;40:60-5.

113. Meyer U, Schwarz MJ, Müller N. Inflammatory processes in schizophrenia: a promising neuroimmunological target for the treatment of negative/cognitive symptoms and beyond. Pharmacol Ther 2011;132:96-110.

114. Cheung C, Yu K, Fung G, Leung M, Wong C, et al. Autistic disorders and schizophrenia: related or remote? An anatomical likelihood estimation. PLoS One 2010;5:e12233.

115. Rowland LM, Pradhan S, Korenic S, Wijtenburg SA, Hong LE, et al. Elevated brain lactate in schizophrenia: a 7 T magnetic resonance spectroscopy study. Transl Psychiatry 2016;6:e967.

116. Fryer SL, Roach BJ, Ford JM, Turner JA, van Erp TG, et al. Relating intrinsic low-frequency BOLD cortical oscillations to cognition in schizophrenia. Neuropsychopharmacology 2015;40:2705-14.

117. Takata A, Ionita-Laza I, Gogos JA, Xu B, Karayiorgou M. De novo synonymous mutations in regulatory elements contribute to the genetic etiology of autism and schizophrenia. Neuron 2016;89:940-7.

118. Kirov G. CNVs in neuropsychiatric disorders. Hum Mol Genet 2015;24:R45-9.

119. Xing J, Kimura H, Wang C, Ishizuka K, Kushima I, et al. Resequencing and association analysis of six PSD-95-related genes as possible susceptibility genes for schizophrenia and autism spectrum disorders. Sci Rep 2016;6:27491.

120. Kasem E, Kurihara T, Tabuchi K. Neurexins and neuropsychiatric disorders. Neurosci Res 2018;127:53-60.

121. Khanzada NS, Butler MG, Manzardo AM. GeneAnalytics pathway analysis and genetic overlap among autism spectrum disorder, bipolar disorder and schizophrenia. Int J Mol Sci 2017; doi: 10.3390/ijms18030527.

122. Schmidt RJ, Tancredi DJ, Ozonoff S, Hansen RL, Hartiala J, et al. Maternal periconceptional folic acid intake and risk of autism spectrum disorders and developmental delay in the CHARGE (CHildhood Autism Risks from Genetics and Environment) case-control study. Am J Clin Nutr 2012;96:80-9.

123. Malkova NV, Yu CZ, Hsiao EY, Moore MJ, Patterson PH. Maternal immune activation yields offspring displaying mouse versions of the three core symptoms of autism. Brain Behav Immun 2012;26:607-16.

124. Morris JA. Schizophrenia, bacterial toxins and the genetics of redundancy. Med hypotheses 1996;46:362-6.

125. Meyer U, Schwendener S, Feldon J, Yee BK. Prenatal and postnatal maternal contributions in the infection model of schizophrenia. Exp Brain Res 2006;173:243-57.

126. van der Ven E Selten JP. Migrant and ethnic minority status as risk indicators for schizophrenia: new findings. Curr Opin Psychiatry 2018;31:231-6.

127. Egerton A, Howes OD, Houle S, McKenzie K, Valmaggia LR, et al. Elevated striatal dopamine function in immigrants and their children: a risk mechanism for psychosis. Schizophr Bull 2017;43:293-301.

128. Ohi K, Shimada T, Yasuyama T, Uehara T, Kawasaki Y. Variability of 128 schizophrenia-associated gene variants across distinct ethnic populations. Transl Psychiatry 2017; doi: 10.1038/tp.2016.260.

129. Eack SM, Bahorik AL, Newhill CE, Neighbors HW, Davis LE. Interviewer-perceived honesty as a mediator of racial disparities in the diagnosis of schizophrenia. Psychiatr Serv 2012;63:875-80.

130. Pinto R, Ashworth M, Jones R. Schizophrenia in black caribbeans living in the UK: an exploration of underlying causes of the high incidence rate. Br J Gen Pract 2008;58:429-34.

131. Hamilton JE, Heads AM, Meyer TD, Desai PV, Okusaga OO, et al. Ethnic differences in the diagnosis of schizophrenia and mood disorders during admission to an academic safety-net psychiatric hospital. Psychiatry Res 2018;267:160-7.

132. Vassos E, Di Forti M, Coleman J, lyegbe C, Prata D, et al. An examination of polygenic score risk prediction in individuals with first-episode psychosis. Biol Psychiatry 2017;81:470-7.

133. Olde Loohuis LM, Mangul S, Ori APS, Jospin G, Koslicki D, et al. Transcriptome analysis in whole blood reveals increased microbial diversity in schizophrenia. Transl Psychiatry 2018;8:96.

134. Sanders AR, Drigalenko EI, Duan J, Moy W, Freda J, et al. Transcriptome sequencing study implicates immune-related genes differentially expressed in schizophrenia: new data and a meta-analysis. Transl Psychiatry 2017; doi: 10.1038/tp.2017.47.

135. Chestkov IV1, Jestkova EM2, Ershova ES1, Golimbet VE3, Lezheiko TV, et al. Abundance of ribosomal RNA gene copies in the genomes of schizophrenia patients. Schizophr Res 2018; doi: 10.1016/j.schres.2018.01.001.

136. Brennand KJ, Simone A, Jou J, Gelboin-Burkhart C, Tran N, et al. Modelling schizophrenia using human induced pluripotent stem cells. Nature 2011;473:221-5. 